\title{
Investigation of the Efficiency of Manufacturing Polymer Molds by FDM-Printing
}

\author{
Oleg Krupennikov ${ }^{1, a}$, Mihail Nazarov ${ }^{1, b}$ and Evgeniy Kiselev ${ }^{1, c^{*}}$ \\ ${ }^{1}$ Ulyanovsk State Technical University, Ulyanovsk, Russia \\ a krupennikov_oleg@mail.ru, ${ }^{b}$ nazarov.mv@inbox.ru, ${ }^{c}$ kec.ulstu@mail.ru
}

Keywords: Additive Technologies, 3D-Printing, Fused Deposition Modeling (FDM), Polymeric Materials

\begin{abstract}
The results of experimental studies of the efficiency of manufacturing polymer foundry models by the FDM method are presented. Rational consumables for 3D-printing are revealed. The optimal values of the shrinkage value of the 3D-model during printing and the parameters of the 3D-printer operation mode are revealed. It has been established that polymer casting molds are rationally manufactured by FDM printing, using ABS-plastic as a consumable, while making a correction to linear dimensions at the stage of creating a 3D-model to eliminate plastic shrinkage.
\end{abstract}

\section{Introduction}

The use of additive technologies in foundry is a promising direction, since over the past few years the cost of 3D-printers has significantly decreased, the print quality has improved and the technology of printing parts of technological equipment has been simplified [1-5].

Currently, several varieties of 3D-printing are used to prototype parts of foundry equipment. At the same time, each of the methods of 3D-printing has a number of advantages and disadvantages that determine the suitability of its use for prototyping various parts of technological equipment.

For example, the method of layer-by-layer deposition of FDM, based on extrusion through a calibrated nozzle of heated and molten fiery thermoplastic, is characterized by the relative cheapness of the equipment and consumables used, as well as high printing speed while ensuring the strength characteristics of the products. At the same time, due to the shrinkage of thermoplastic during its cooling, warping and delamination of parts can occur, which, in turn, can lead to a decrease in the accuracy of their geometric parameters and surface quality.

To assess the effectiveness of the manufacture of polymer casting molds by FDM-printing, the authors conducted a number of experimental studies.

The experiments were carried out by printing on the FDM-printer Anycubic i3 Mega S STLmodels of parts of casting molds, pre-processed in the Ultimaker Cura slyser.

In order to find out how the dimensions of parts change in the process of 3D-printing due to plastic shrinkage, at the first stage of research samples were printed in the form of a parallelepiped with dimensions of $120 * 15 * 10 \mathrm{~mm}$ from four types of plastic: ABS, PLA, PETG and SBS. The obtained samples measured the actual overall dimensions and compared with similar dimensions set in the 3D-model of the sample during printing.

It is established that the shrinkage of plastic, regardless of its brand, is significantly influenced by the value of the nominal value of the overall size. In this case, the lowest shrinkage is recorded along the length of L samples, and the highest shrinkage value is obtained by the height of $\mathrm{H}$ samples. In addition, the amount of shrinkage of the samples is influenced by the degree of plastic filling of the 3D-model, which was installed in the 3D-model of the sample during the preparation for printing and ranged from $40 \%$ to $100 \%$. 
It was revealed (fig.1) that with an increase in the degree of filling the model with plastic, there is a slight change in the shrinkage value on the one hand, and on the other hand, as the degree of filling the sample with plastic increases, there is an increase in shrinkage fluctuations in samples made of different materials. Obviously, a large amount of plastic filling the sample during the printing process leads to large temperature deformations and fluctuations in the properties of plastics.

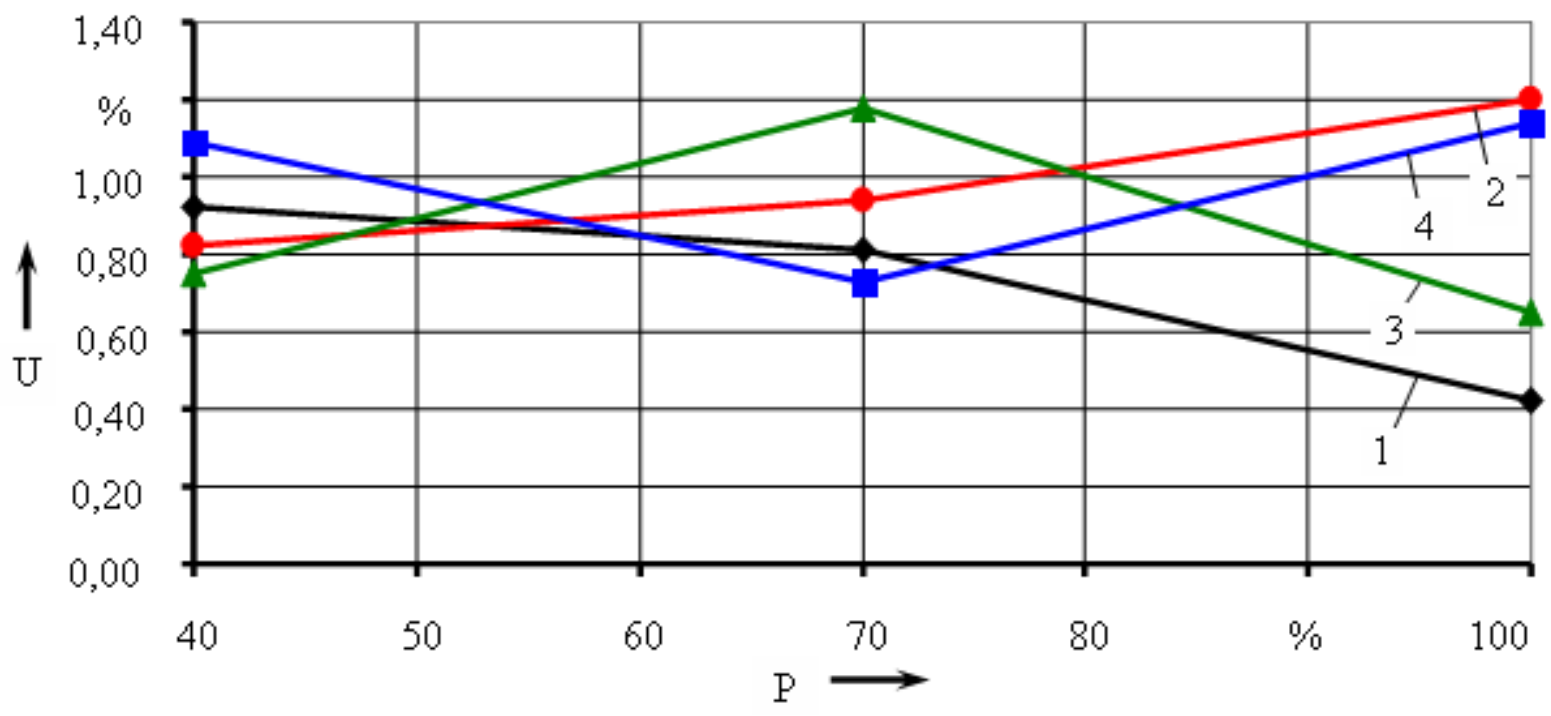

Fig. 1. Effect of the degree of filling of the 3D-model $P$ on the shrinkage of $U$ samples from the studied plastics: 1,2,3,4 - respectively ABS-, PLA-, PETG - and SBS-plastic

In the process of research, it was established (fig. 2) that the average shrinkage value with an increase in the degree of filling the sample with plastic decreases from $0.9 \%$ to $0.7 \%$.

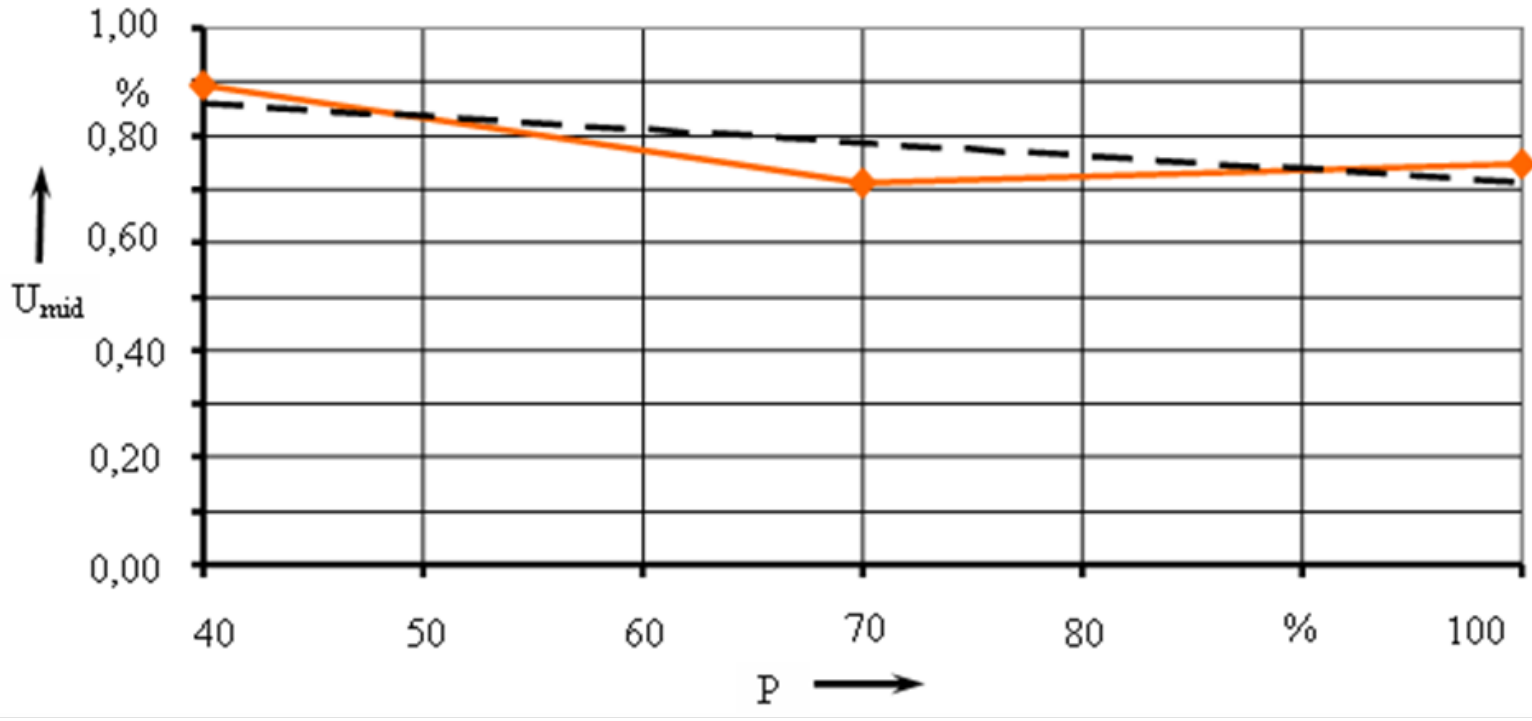

Fig. 2. Effect of the degree of filling of the 3D-model $P$ on the average shrinkage $U_{\text {mid }}$ samples from the studied plastics 
Thus, when developing 3D-models of casting mold parts for the manufacture of wax blanks, it is necessary to make a clarification equal to $1.07-1.09$, depending on the degree of filling of the model.

In accordance with the developed methodology, at the second stage of research, rational materials were selected for 3D-printing of parts of foundry molds. Varied four plastic materials (ABS, PLA, PETG and SBS) under constant printing modes corresponding to the recommended values from the reference literature.

As can be seen from figure 3, the lowest values of the height parameters of the surface profile are achieved in samples made of ABS-plastic, slightly worse indicators in samples from PLA- and PETG-plastics. The worst parameters were shown by samples made of SBS-plastic.

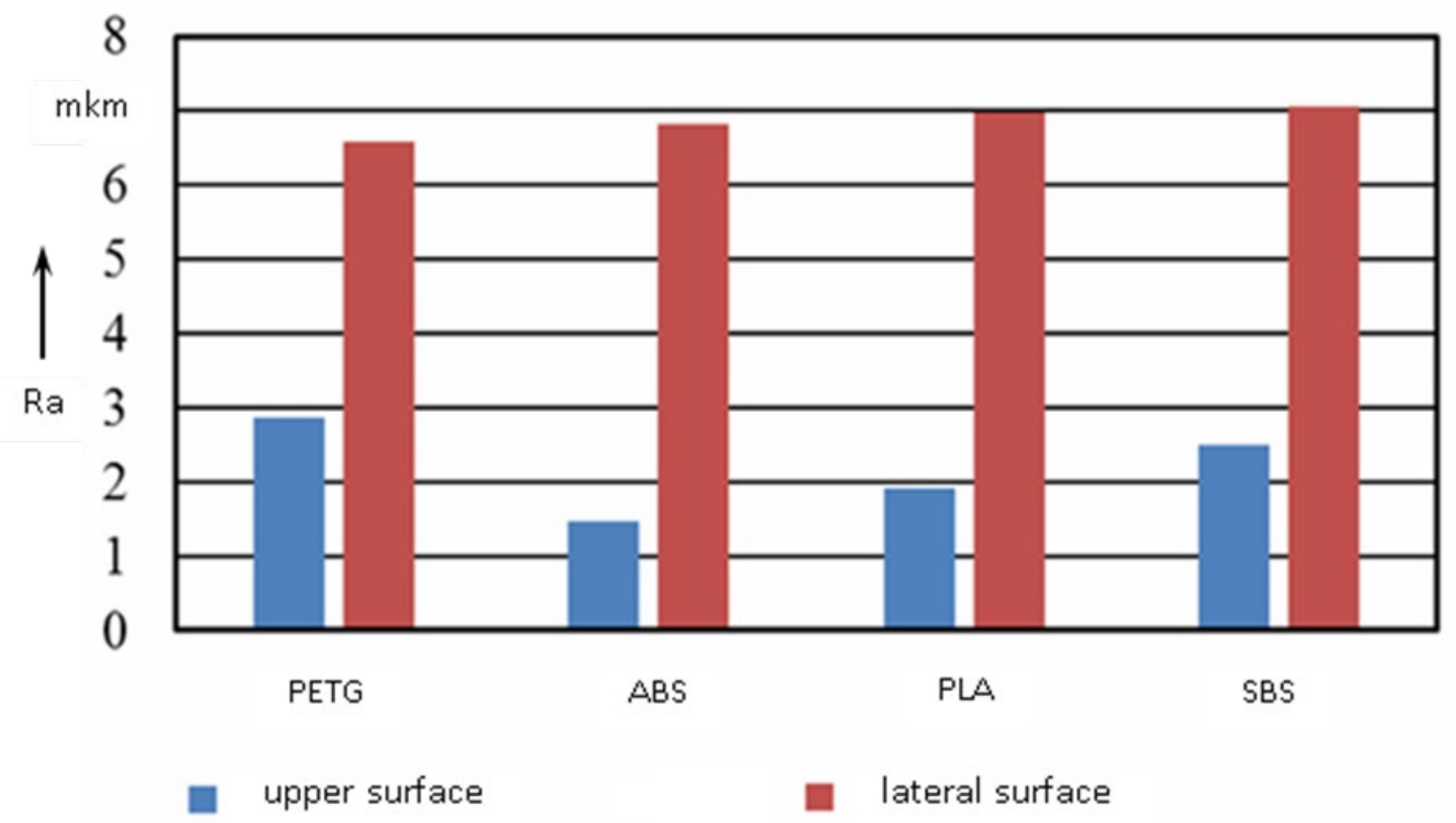

Fig. 3. Influence of plastic material on the arithmetic mean deviation of the surface profile $\mathrm{Ra}$

In order to draw definitive conclusions in favor of choosing a rational material for 3D-printing of casting mold parts, studies were conducted to determine the toughness of the samples.

As can be seen from the results presented in figure 4, samples made of ABS-plastic have the greatest toughness. PETG-plastic samples have a toughness (10-15) \% less than that of ABSplastic samples. The worst result was shown by samples from PLA- and SBS-plastics - their toughness is (40-50) \% less than that of samples made of ABS-plastic. Thus, the best quality of 3D-printing is provided by this indicator in samples printed from ABS- and PETG-plastics, which have the highest toughness.

However, the cost of PETG-plastic is 1.4-1.5 times higher than ABS-plastic with the same degree of shrinkage of the material. Therefore, based on the results of experimental studies, ABSplastic should be recommended as a rational material for 3D printing of casting mold parts. 


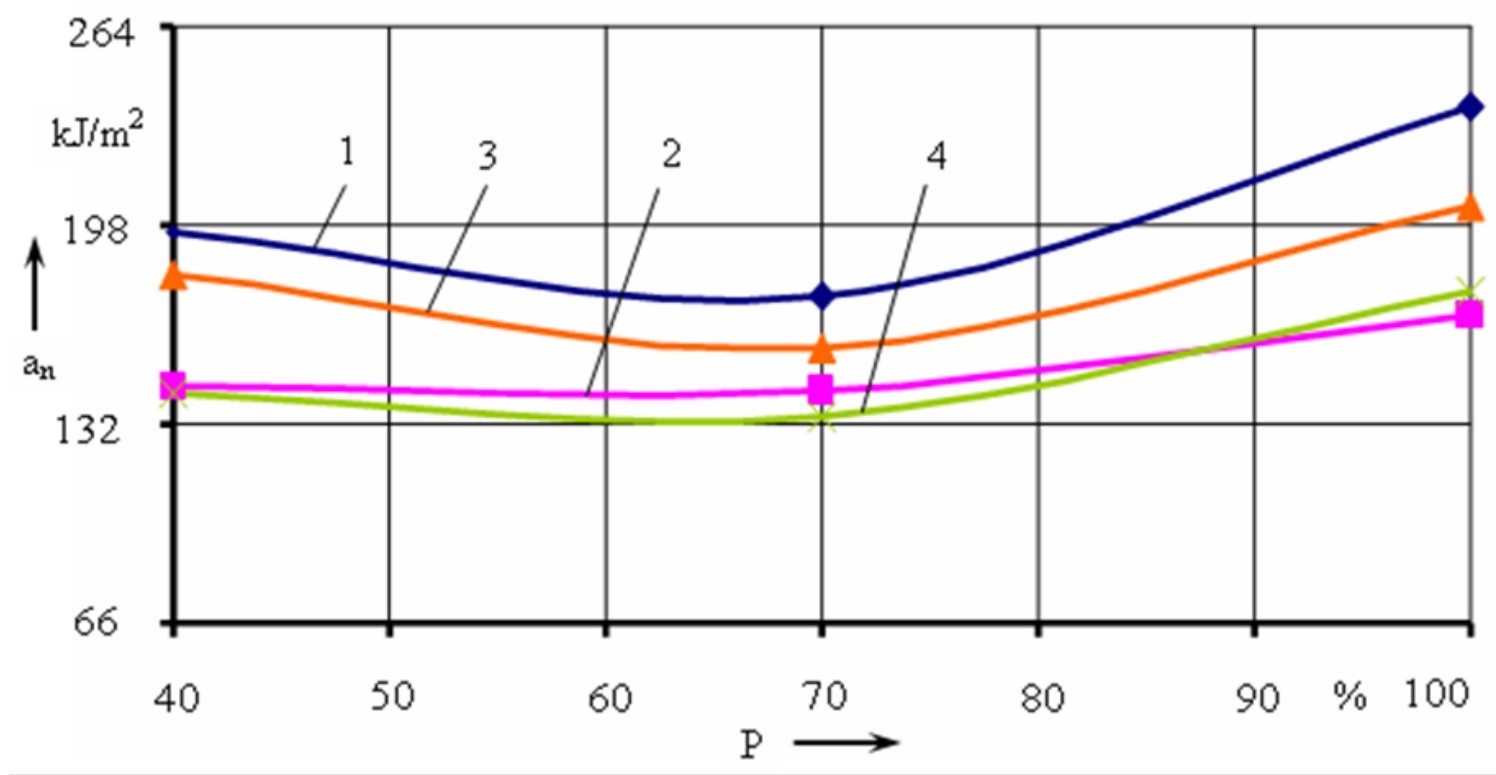

Fig. 4. Effect of the degree of filling of the $3 D$-model $P$ on the toughness $a_{n}$ samples depending on the grade of material: 1, 2, 3, 4-respectively ABS-, PLA-, PETG-and

\section{SBS-plastics}

Increasing the degree of filling P to $100 \%$ leads to an increase in the toughness of an samples by (15-20) \% (see figure 4). Therefore, for the manufacture of parts of casting molds with increased toughness, they must be printed at $100 \%$ filling of the 3D-model.

In the third stage of the research, the influence of the elements of the 3D-printing mode of casting mold parts on the efficiency of the printing process was evaluated. The samples were made of ABS-plastic, chosen as a rational material in the second stage of research.

It was found that the increase in the temperature of the extruder slightly affects the altitude parameters of the irregularities of the upper and lateral planes of the samples. In this regard, $\mathrm{Te}=$ $240{ }^{\circ} \mathrm{C}$ was taken as the recommended temperature of the extruder nozzle, which corresponds to the recommended reference data.

In the course of research, it was revealed that small values of the temperature of the 3D-printer table in the range (100-110) ${ }^{\circ} \mathrm{C}$ lead to significant fluctuations in the values of the altitude parameters of the surfaces of the printed sample. This indicates unfavorable conditions for the formation and subsequent cooling of the layers of the product. With a further increase in the table temperature to $120^{\circ} \mathrm{C}$, the print quality stabilizes, which indicates an exit to the rational mode of 3D-printing.

In studies of the influence of the extruder displacement Ve on the efficiency of 3D-printing, it was established (see figure 5) that the Ve velocity has significant changes in the roughness (according to the Ra parameter) of the upper and lateral surfaces of the samples under study.

As can be seen from the results of the studies presented in figure 5, it is most rational to move the extruder in the process of 3D-printing at a speed of (35-40) $\mathrm{mm} / \mathrm{s}$. When the specified limit is exceeded, the roughness parameter $\mathrm{Ra}$ of the lateral plane of the samples increases significantly, which indicates that the plastic does not have time to solidifies and it is deformed by the newly applied layer of molten filament. 


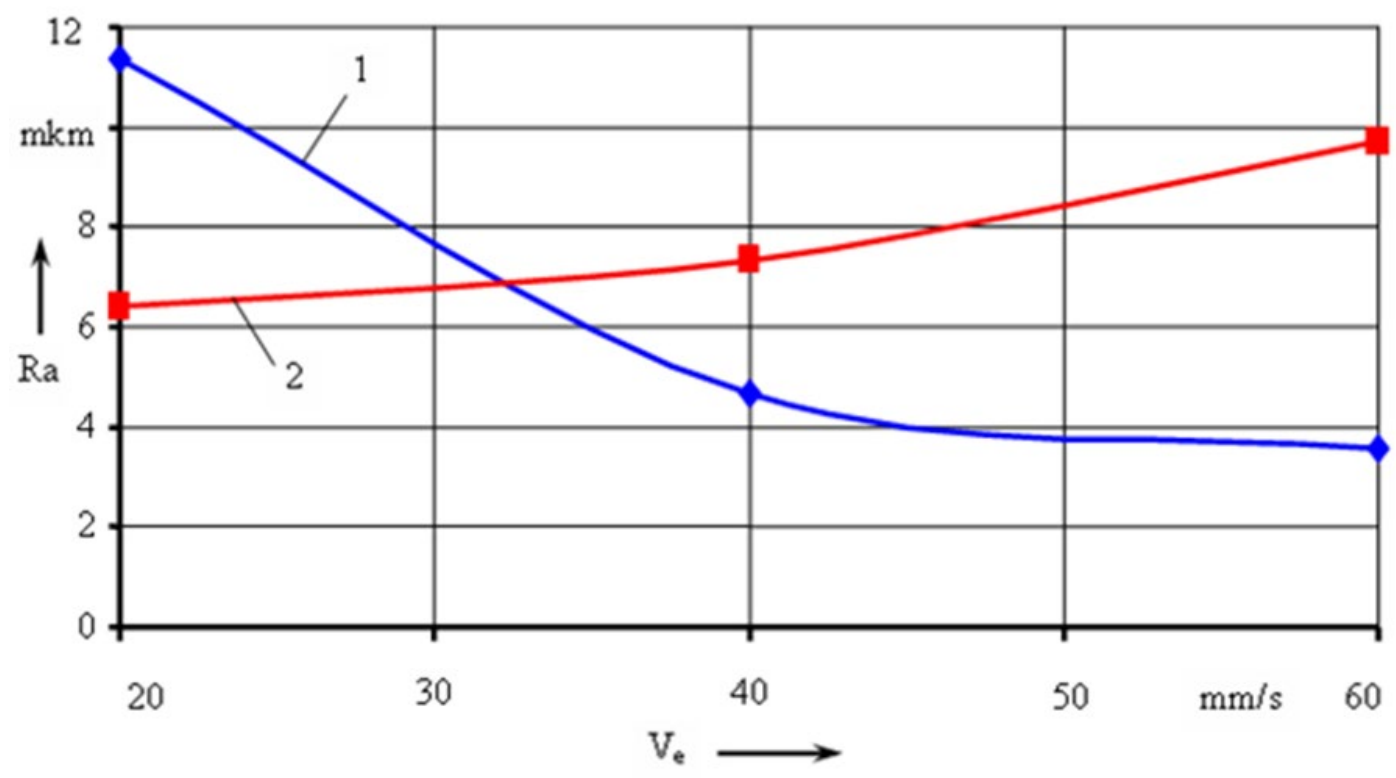

Fig. 5. Influence of the velocity of the extruder $V_{e}$ on the arithmetic mean deviation of the surface profile Ra: 1 and 2 - respectively the upper and lateral planes of the sample

Thus, experimental studies have shown that polymer casting molds are rationally made by FDM-printing, using ABS-plastic as a consumable, making correction to linear dimensions at the stage of creating a 3D-model to eliminate shrinkage of plastic.

\section{References}

[1] Singh, S. Precision investment casting: A state of art review and future trends / S. Singh, R. Singh // Proceedings of the Institution of Mechanical Engineers, Part B: Journal of Engineering Manufacture.- 2016. - T. 230. - № 12. - C. 2143-2164.

https://doi.org/10.1177/0954405415597844

[2] Bourell, D.L. Roadmap for additive manufacturing: identifying the future of freeform processing / D.L. Bourell, M.C. Leu, D.W. Rosen. - The University of Texas at Austin, 2009. C. 11-15.

[3] Harun, W.S.W. Characteristic studies of collapsibility of ABS patterns produced from FDM for investment casting / W.S.W. Harun [et al.] // Materials Research Innovations. - 2009. - T. 13. - № 3. - C. 340-343. https://doi.org/10.1179/143307509X441513

[4] Piekło, J. Methods of Additive Manufacturing used in the Technology of Skeleton Castings / J. Piekło, M. Maj // Archives of Metallurgy and Materials. - 2014. - T. 59. - № 2. - C. 699-702. https://doi.org/10.2478/amm-2014-0114

[5] Olkhovik, E.O. Use of additive technologies for practical working with complex models for foundry technologies / E.O. Olkhovik, A.A. Butsanets, A.A. Ageeva // IOP Conference Series: Materials Science and Engineering. - IOP Publishing, 2016. - T. 140. - № 1. https://doi.org/10.1088/1757-899X/140/1/012013 